(C) 2015 IEEE. Personal use of this material is permitted. Permission from IEEE must be obtained for all other uses, in any current or future media, including reprinting/republishing this material for advertising or promotional purposes, creating new collective works, for resale or redistribution to servers or lists, or reuse of any copyrighted component of this work in other works 


\title{
Designing Adaptive Integral Sliding Mode Control for Heart Rate Regulation During Cycle-Ergometer Exercise Using Bio-feedback
}

\author{
Ahmadreza Argha, Steven W. Su, Hung Nguyen and Branko G. Celler ${ }^{\star}$, Fellow, IEEE
}

\begin{abstract}
This paper considers our developed control system which aims to regulate the exercising subjects' heart rate (HR) to a predefined profile. The controller would be an adaptive integral sliding mode controller. Here it is assumed that the controller commands are interpreted as biofeedback auditory commands. These commands can be heard and implemented by the exercising subject as a part of the control-loop. However, transmitting a feedback signal while the pedals are not in the appropriate position to efficiently exert force may lead to a cognitive disengagement of the user from the feedback controller. To address this problem this paper will employ a different form of control system regarding as "actuator-based event-driven control system". This paper will claim that the developed event-driven controller makes it possible to effectively regulate HR to a predetermined HR profile.
\end{abstract}

\section{INTRODUCTION}

Different methods have been introduced for HR regulation during treadmill exercises such as, e.g. classical proportional, integral and derivative (PID) control [1], $\mathrm{H}_{\infty}$ control [2], [3] and model predictive control [4]. In this case, the treadmill speed and/or treadmill gradient are controlled directly by the controller. This problem has been studied for the cycle-ergometer exercises by adjusting the resistance of the brake rather than the pedaling rate to change the exercise level [5]. However, this requires an externally controllable automatic braked cycle-ergometer. Again, the control system controls the load on the cycling system by changing the resistance of the brake device. However, computer controlled cycle-ergometers are very expensive and are not suitable for use at home as a rehabilitation device. Furthermore, our experience is that patients find increasing workload by increasing the pedalling rate more acceptable than increasing workloads at a constant pedalling rate by increasing the braking effect. Alternatively, one may resort to convert the controller commands to signals that could be realized by the exercising subjects. Then the human operator assumes the role of actuator of the control system. These signals commonly referred to as biofeedback. This novel framework could obviate cycle-ergometers from the expensive controllable automatic brake equipment.

Now, a periodic auditory signal would be utilized to motivate the exercising subject to vary their exercising rate. In other words, a translating function, in accordance with the level of the controller output signal, changes the rate

A. Argha, S. W. Su and Hung Nguyen are with Faculty of Engineering and Information Technology, University of Technology, Sydney, PO Box 123, Broadway, NSW 2007, Australia

* B. G. Celler is currently the principal scientist in the division of computational informatics, CSIRO, Marsfield, NSW 2122, Australia Branko.celler@csiro.au

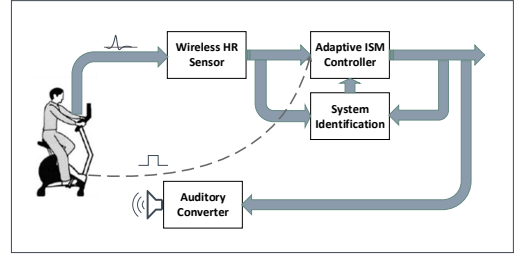

Fig. 1. Block diagram for the HR regulation system during cycling exercise

of auditory stimulus per minute to stimulate the exercising subject to vary their exercising rate. However, this simple scheme ignores the position of subjects' feet in the control system design. This results in a control system referred to as time-driven control systems, which implements the control calculations all the time at a fixed rate. Nevertheless, this may also set the stage for the cognitive disengagement of the subject from the feedback controller. To address this problem, we adjust the time duration of the auditory stimulus and/or the frequency of auditory stimulus instead. Now the rate of transmitting the auditory stimulus to the exercising subjects could be synchronized with the rate of pedalling. This new idea, however, requires a non-conventional control system strategy (see Fig. 1), regarded here as actuator-based event-driven control system, which is a different counterpart of the existing sensor-based event-driven control system in the literature [7].

HR response to dynamic exercise is nonlinear and may also vary for every exercising subject, in different physical situations [6]. Therefore, an adaptive and/or model-based control strategy might normally be exploited to address the problem of HR regulation. In other words, to use the non-model-based control schemes such as proportional, integral and derivative (PID) control the proper settings to be used for the controller parameters should be determined continuously. To cope with the time-varying nature of the HR response (for different subjects), here, an adaptive integral sliding mode control strategy is proposed utilizing online system identification idea. Sliding mode control (SMC) is a well-known robust control method thanks to its ease of design and robustness against matched disturbances. However, traditional SMC would result in the deadbeat control which is undesirable in practical control systems due to the possible large control efforts. Alternatively, the integral sliding mode control (ISMC) design avoids the deadbeat response by removing the poles at zero [8]. In this paper, an event-driven adaptive integral sliding mode 
control is proposed, in which in order to deal with the probable inaccurate controller parameters and/or individual HR profile, two anti-windup mechanisms are designed to protect the user against possible big HR fluctuations.

\section{CONTROL SYSTEM}

\section{A. Model identification}

As it was stated, since the HR response to dynamic exercise is time-varying, a stationary control scheme may not provide adequate performance. Therefore here an adaptive control scheme would be utilized to deal with the timevarying dynamics. To this end, firstly, we consider an linear time-varying (LTV) system to model the human HR response to the control inputs. The model input here is the control commands that the controller generates. The relationship between the input and output is represented as:

$$
\begin{aligned}
y(k)= & a_{1, i} y(k-1)+\cdots+a_{n, i} y(k-n) \\
& +b_{1, i} u(k-1)+\cdots+b_{m, i} u(k-m),
\end{aligned}
$$

where $y(k)$ and $u(k)$ are the system output (HR) and system input (controller commands), respectively and $a_{j, i}$ and $b_{j, i}$ denote the time varying parameters ( $i$ is used here to show that these parameters are time-varying but not with the same rate of $k$ ). The above model can describe a variety of systems. For example, assuming the system to be first order we have:

$$
y(k)=a_{i} y(k-1)+b_{i} u(k-1) .
$$

Now, at the first step, a constant input is provided for the system. The HR is then measured, i.e. every $10 \mathrm{sec}$. After a while i.e. $30 \mathrm{sec}$, the time-varying parameters for LTV model in (1) (we assumed a first order system) are identified using the well-known Kalman filter (KF) approach.

\section{B. Discrete-time ISMC}

Consider the following system which is derived from the transfer function in (1),

$$
x(k+1)=a_{i} x(k)+b_{i} u(k)+d(k),
$$

where $x(k)$ denotes the HR $(y(k)$ in $(1)), u(k)$ is the control input, $d(k)$ denotes the disturbances and model uncertainties which is assumed to be bounded as $|d(k)|<\bar{d}(\bar{d}>0$ is a known scalar), $a_{i}$ and $b_{i}$ are the time varying system parameters which are scalars. Now the integral sliding surface is proposed as

$$
\begin{aligned}
& \sigma(k)=e(k)-e(0)+v(k) \\
& v(k)=v(k-1)+\gamma e(k-1),
\end{aligned}
$$

where $e(k)=r(k)-x(k)$ denotes the tracking error $(r(k)$ is the reference trajectory), $\sigma(k), v(k)$ are the sliding function and integral variable and $\gamma$ is the design parameter. Now letting $\sigma(k+1)=0$ (which is a routine in sliding mode control) would result in

$$
\begin{aligned}
\sigma(k+1) & =e(k+1)+v(k+1)-e(0) \\
& =e(k+1)-(1-\gamma) e(k)+v(k)+e(k)-e(0) \\
& =e(k+1)-(1-\gamma) e(k)+\sigma(k)=0
\end{aligned}
$$

Hence utilizing (2) one may show that the so-called equivalent controller is

$$
u_{e q}(k)=b_{i}^{-1}\left[r(k+1)-\eta e(k)-a_{i} x(k)-d(k)+\sigma(k)\right],
$$

where $\eta=1-\gamma$. However, it is not possible to implement the above controller as $d(k)$ is unknown. Since in this work a moving average filter is utilized for smoothing the HR signal and also the rate of updating the system model is very slower than the sampling rate, we can use the disturbance estimate in the controller as

$$
u(k)=b_{i}^{-1}\left[r(k+1)-\eta e(k)-a_{i} x(k)-\hat{d}(k)+\sigma(k)\right],
$$

where $\hat{d}(k)$ denotes the disturbance estimate which can be achieved by the following disturbance estimator,

$$
\hat{d}(k)=d(k-1)=x(k)-a_{i} x(k-1)-b_{i} u(k-1) .
$$

\section{Stability analysis}

As a result of applying the controller to the system (2), we have

$$
x(k+1)=r(k+1)-\eta e(k)+d(k)-\hat{d}(k)+\sigma(k),
$$

and knowing that $\sigma(k)=\hat{d}(k-1)-d(k-1)$, then

$$
e(k+1)=\eta e(k)+\beta(k),
$$

in which $\beta(k)=d(k)-2 d(k-1)+d(k-2)=\mathrm{O}\left(T^{3}\right)$ (see [8]). Since $\eta=1-\gamma$, one can choose $\gamma$ such that $\eta<1$. This suffices that the tracking error dynamics remains bounded as $\beta(k)$ is bounded as well.

\section{Actuator-based Event-driven Control Systems}

The sampling rate of the HR transmitted to the program by an ecg or pulse oximeter transducer is typically constant (1 sample per second), however, the rate of sending the commands to the exercising subject varies according to the pedaling rate.This form of control system, called here actuator-based event-driven control system, is obviously different from the traditional control systems. Possibly, it could be considered somewhat as multi-rate systems, as the rates of output sampling and control input updating are different, however, since the updating rate of control input varies according to the pedaling rate of the exerciser, it could be also assumed to be an event-based control system. Nevertheless, our proposed system is not a sensor-based event-driven one; see [7]. It is because of this that the aim of this control system is to control the exercise rate through heart rate and not to control the velocity or the position of a rotary system (DC motor in the aforementioned control systems and pedaling system here) and the HR sensor signal, sampled with a constant rate independent from the pedaling rate, would not trigger the control actions. Hence, the methods considered for the sensor-based event-driven control systems, which uses the spatial domain of the system model [7], could not be adapted to our work.

Notice that due to the fixed sampling rate of the HR sensor, in the case that more than one update is required during each sample period, no new HR value, and hence, tracking error 
is available for the second and later updates. As a result, the controller can only be updated using the last available information. Consequently, it could be more likely to suffer from the windup phenomena in the system performance.

In this paper, we utilize a time-driven ISMC in the form which is given in the previous subsection, assuming a fixed sampling rate $T$ equal to the sampling period of the HR sensor. Then, whenever an event occurs (the left or right pedal pass the reed sensor), the last prepared update will be transmitted as a biofeedback signal. Since the rate of transmitting the biofeedback is synchronized by the pedaling rate (events), this method is called event-driven method here.

\section{E. HR profile}

The HR profile, in this work, is selected in three stages. In the first stage, called the warm up period, we aim to gradually increase the user HR from their normal HR $\left(H R_{n}\right)$ to the exercise $\mathrm{HR}\left(H R_{e}\right)$ which then remains constant for a while $\left(T_{e}\right)$. The warm up slope is

$$
\theta_{w}=\frac{H R_{e}-H R_{n}}{T_{w}},
$$

in which $\theta_{w}$ represents warm up slope and $T_{w}$ shows the warm up time duration. The warm up time duration $T_{w}$ is indeed used to avoid an excessively rapid increase in the HR. The second stage called the exercise period is indicated by $T_{e}$, which may be computed according to the maximum HR. The maximum HR $\left(H R_{\max }\right)$ commonly is characterized by the well-known Haskell and Fox formula as:

$$
H R_{\max }=220-\text { age }
$$

We set then the exercising $H R_{e}$ as $0.65 \sim 0.85$ of $H R_{\max }$, depending on the subjects level of fitness and/or risk profile. The application of the third cooling down or recovery stage $\left(T_{c}\right)$ is basically to prevent possible venous pooling and reduced venous return to the heart from an excessively abrupt termination in exercise.

\section{F. Auditory convertor}

As mentioned, in order of facilitating the user applying more or less effort at the right time, it is found that the combination of varying both the time duration and the frequency is an effective method.

In this method a longer time duration and lower frequency is used to motivate the subject to do the pedalling slowly and vice versa at the quick pedaling. Moreover, the range of time duration and frequency which can be used are limited. We know that the time durations more than a specific value result in the so-called aliasing phenomena. Additionally, the time durations which are less than a specific value may not be heard by the subjects. This issue also holds for the auditory signals' frequency. As a result, we selected the following range of time duration and frequency for the auditory stimulus as $60 \sim 500 \mathrm{~ms}$ and $400 \sim 2000 \mathrm{~Hz}$, respectively. Therefore, the auditory converter contains two saturation functions,

$$
t_{d}=\operatorname{sat}\{g(u(k))\}=\left\{\begin{array}{lr}
\lambda_{1} & g(u(k)) \leq \lambda_{1} \\
g(u(k)) & \lambda_{1}<g(u(k))<\lambda_{2} \\
\lambda_{2} & g(u(k)) \geq \lambda_{2},
\end{array}\right.
$$

where $t_{d}$ is the time duration of the auditory signals and $g(u(k))$ is a scaling function which converts the range of $u(k)$ to the range of time duration, $\lambda_{1}=60 \mathrm{~ms}, \lambda_{2}=500 \mathrm{~ms}$, and

$$
f_{b}=\operatorname{sat}\{h(u(k))\}=\left\{\begin{array}{lr}
\gamma_{1} & h(u(k)) \leq \gamma_{1} \\
h(u(k)) & \gamma_{1}<h(u(k))<\gamma_{2} \\
\gamma_{2} & h(u(k)) \geq \gamma_{2}
\end{array}\right.
$$

where $f_{b}$ is the frequency of the auditory signals and $h(u(k))$ is also a scaling function which converts the range of $u(k)$ to the range of appropriate frequencies of the auditory signal, $\gamma_{1}=400 \mathrm{~Hz}$ and $\gamma_{2}=2000 \mathrm{~Hz}$

\section{G. Implementing anti-windup mechanisms}

The so-called windup phenomena may happen due to the utilizing the integral action in the controller and saturation in the system actuators. Here, as the exercising subject plays the role of system actuator, and has its exercise limitations, windup may occur. Indeed, during the windup phenomena the closed-loop control system cannot implement its correct control strategy and the actuators will perform at their limits independently of the system outputs measured. In the case of utilizing an integrator in the controller, the error will be integrated continuously for a long period before things return to normal. Hence, control effort may increase to a very large value. Thus the ISM controller may cause large transients while the system actuator saturates.

If the exercising subject inadvertently or deliberately chooses not to follow the controller commands, a big tracking error will be generated, and the controller will require a huge effort to compensate for the absent effort. This may result in a big fluctuation in the $\mathrm{HR}$ of the subjects. If HR profile (such as warm up slope $w_{s}$ in (7) and target HR $\left.\left(H R_{e}\right)\right)$ is not determined accurately for each subject, considering the maximum work-rate that the subject can do whilst pedaling continuously, a collapse in the closed-loop mechanism could occur. Even if the target HR and warm up slope are set appropriately according to each subject's condition, the subject may deliberately choose to stop the exercise for a short while.

In the conventional control technology, to deal with this problem, a number of anti-windup schemes have been developed, which need to know and use the actuator limitations. Here, however, the exercising subjects activity limitations are not easily obtained. Furthermore, it is not always possible to determine that the subject's unresponsiveness is inadvertent or deliberate. In order to address the problem, the HR profile needs to be more dynamic. In other words, in the cases that big tracking errors are noted (bigger than a specific threshold), the HR profile could be temporarily held constant to prevent big efforts being required of the subjects. We thus 
revise the HR profile as follows,

$$
w_{s}= \begin{cases}\frac{H R_{e}-H R_{n}}{T_{w}} & \text { if } e(k) \leq \delta, \\ 0 & \text { if } e(k)>\delta,\end{cases}
$$

where $\delta>0$ denotes a certain threshold. Notice that the warm up duration will then increase to $\bar{T}_{w}=T_{w}+T_{a}$ which $T_{a}$ is the summation of all time periods that $e(t)>\delta$ and $w_{s}=0$. This could avoid any big fluctuation in the HR of the subject.

Since the designed control system contains an auditory converter (biofeedback generator) it is also possible that both converters (audio frequency and time duration) reach their limitations, even with accurate definition of the translating functions $t_{d}$ and $f_{b}$ in (9) and (10), respectively. This could also result in windup phenomena. Here, the windup would be addressed by using $u_{a w}(k)$ instead of $u(k)$, where

$$
\left\{\begin{array}{l}
u_{a w}(k)=u_{a w}(k-1), \quad \text { if }\left\{\begin{array}{l}
\lambda_{1}<g(u(k))<\lambda_{2} \\
\gamma_{1}<h(u(k))<\gamma_{2}
\end{array}\right. \\
u_{a w}(k)=u(k), \\
\text { otherwise. }
\end{array}\right.
$$

\section{Methodology}

In this project, we utilized a Nonin 4100 Pulse Oximeter to collect the HR data during the exercise. Besides, the cycleergometer that we used in this work is an air vane exercise bike, which is especially well adapted for safe exercise by the frail elderly. Since it has a big supporting structure, which can be used by the exercising patient to stabilize themselves during exercising, the finger pulse oximeter is almost completely stable during exercise and generates minimal artefact. We have developed an acquisition system using the National Instrument LabVIEW which provides easy synchronization and graphical user interface.

In addition, we attached two reed switches and magnets to the crank shaft of pedals of exercise bike, in order to provide a pulse whenever a full revolution has been completed. In the Labview program, a time-delay parameter may be set to help the user to adjust the time-delay between the position of the pedal sensed by the sensor and the time when the command will be sent. This parameter in the designed software is useful since we can set the point where the user can most effectively apply force to the pedals.

\section{Simulation RESUlts AND EXPERIMENTAL STUDY}

Now in order to check the performance of the proposed controller we consider a numerical example. Consider the system in (2) with 3 different models as

$$
\begin{cases}a_{1}=1.5 & b_{1}=2.0 \text { if } 0 \leq k \leq 30 \mathrm{sec} \\ a_{2}=1.2 & b_{2}=1.3 \text { if } 31 \leq k \leq 80 \mathrm{sec} \\ a_{3}=1.0 & b_{3}=1.4 \text { if } 81 \leq k \leq 150 \mathrm{sec}\end{cases}
$$

with $d(k)=2 \sin \left(\frac{k}{3}\right) \cos \left(\frac{k}{6}\right)$. Assuming $\gamma=0.1$ and hence $\eta=0.9$, the controller in (4) is applied to the system (2). A reference model generates a reference signal $r(k)$ corresponding to a warm up slope to increase HR from 61 $\mathrm{bpm}$ to a steady state value of $140 \mathrm{bpm}$. The controller now aims to regulate the pedaling rate so that the exerciser's HR

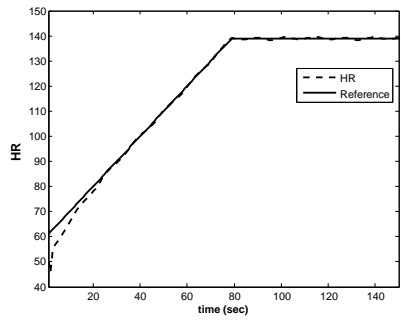

Fig. 2. Simulation result

tracks this reference signal. The result is shown in Fig. 2. Simulation result here shows that the proposed controller has the ability to follow a predefined exercise HR profile in the presence of external and internal disturbances $(d(k))$. In the future, the controller will then be validated by an experimental study, using the designed automated system, that will involve different kinds of reference HR signals. In addition, to improve the performance of the automated system, a higher order model (i.e. second order) will be tried.

\section{CONCLUSIONS}

In this study, a new control system called adaptive actuator-based event driven control system has been designed to help the exercising subjects to track a preset HR profile. The advantage of this study, compared to other published data, is that in our automated system the biofeedback signals are synchronized with respect to the positions of the pedals. However, this required to design a new control scheme instead of the conventional time-driven control systems used in the literature. Implementing a simulation, it has been shown that the proposed controller had the ability to track the HR predetermined profile. Our next step is to validate the controller by an experimental study.

\section{REFERENCES}

[1] T. Kawada, G. Sunagawa, H. Takaki, T. Shishido, H. Miyano, Miyashita, T. Sato, M. Sugimachi and K. Sunagawa, "Development of a servo-controller of heart rate using a treadmill," Japanese Circulation Journal, vol. 63, pp. 945-950, 1999.

[2] T. M. Cheng, A. V. Savkin, B.G. Celler, S. W. Su, and L. Wang, "Nonlinear modeling and control of human heart rate response during exercise with various work load intensities," IEEE Transactions on Biomedical Engineering, vol. 55, no. 11, pp. 2499-2508, 2008.

[3] S. W. Su, L. Wang, B. G. Celler, A. V. Savkin, and Y. Guo, "Identification and control for heart rate regulation during treadmill exercise," IEEE Transactions on Biomedical Engineering, vol. 54, no. 7, pp. 1238-1246, 2007.

[4] S. W. Su, S. Huang, L. Wang, B. G. Celler, A. V. Savkin, Y. Guo, and T. Cheng, "Nonparametric hammerstein model based model predictive control for heart rate regulation," in Proc. IEEE Eng. Med. Biol. Soc., pp. 2984-2987, 2007.

[5] T. Kawada et al, "Development of a servo-controller of heart rate using a cycle ergometer," Heart Vessels, vol. 14, pp. 177-184, 1999.

[6] D. Baig, F. Javed, A. V. Savkin and B. G. Celler, "An Adaptive $\mathrm{H}_{\infty}$ Control Design for Exercise-Independent Human Heart Rate Regulation System," in Proc. the 9th IEEE International Conference on Control and Automation (ICCA), Santiago, Chile, Dec. 19-21, 2011

[7] W. P. M. H. Heemels, J. H. Sandee, and P. P. J. Van Den Bosch, "Analysis of event-driven controllers for linear systems," International Journal of Control, vol. 81, no. 4, pp. 571-590, 2008

[8] K. Abidi, J.X. Xu, "On the discrete-time integral sliding mode control," inProc. IEEE Workshop on Variable Structure Systems VSS 2006, Alghero, Italy, 2006. 